\title{
Never miss- a near miss audit
}

\author{
Abstract \\ Context: Near-miss cases that defines the patients who just escaped death, are a valuable \\ source of scrutiny to describe the causes of maternal death. This in turn can help in \\ estimating the quality of care in the institute and amount of facilities required for a given \\ population.
}

Aim: Evaluating the incidence and nature of near-miss events by calculating the near- miss indicators.

Settings and Design: An observational study.

Methods and Materials: A study of near-miss cases and maternal mortality cases for their demographic profile, causes of the near -miss condition, outcome of the near-miss condition and classification of these cases according to World Health Organization (WHO) criteria.

Results: Among the sixty-four near-miss and mortality cases, fifty-two were referred and only twelve developed potentially life threatening conditions within the hospital. Postpartum hemorrhage was the most common cause of near-miss event $(32 \%)$. Septicemia has the highest case fatality rates. No maternal mortality due to hypertensive disorders of pregnancy.

Conclusion: Prevention is always better than cure but sometimes timely diagnosis intervention is as good as prevention. Hence decreasing the burden of near-miss and maternal mortality is more dependent on active intervention which is possible only if timely referrals are made. Thus the community health workers and other health related personnel must try to do their best to create awareness in the community about pregnancy related complications and the need for timely referrals.

Keywords: Severe acute maternal morbidity, Maternal near-miss indices
Volume 6 Issue I - 2017

\author{
Sapna Vinit Amin, Sindhuja Bala, Kumar P, Rai \\ L, Pai MV, Shetty J \\ Department of Obstetrics \& Gynecology, Manipal University, \\ India
}

Correspondence: Sapna Vinit Amin, Department of Obstetrics \& Gynecology, Kasturba Medical College, Manipal University, Manipal, Karnataka, India, Email drsapnagoni@rediffmail.com

Received: September 13, 2016 | Published: January 12, 2017
Abbreviations: WHO, World Health Organization; SMOR, Severe Maternal Outcome Ratio; MNMR, Maternal Near-Miss Ratio; SMOR, Severe Maternal Outcome Ratio; MI, Mortality Index

\section{Introduction}

Maternal mortality has reduced from 400 per 1,00,000 live births in 1990 to 210 per $1,00,000$ live births in $2010 .{ }^{1}$ This decrease in maternal mortality over the past two decades has been achieved with improving overall medical facilities. However on critically evaluating these cases the underlying cause cannot be made satisfactorily because of less number. Thus auditing maternal near-miss cases, which occurs one step before mortality, gives an idea about the trend of maternal mortality. It also reflects the quality of care a patient receives in the institute. Maternal near miss case is defined as "a woman who nearly died but survived a complication that occurred during pregnancy, childbirth, or within 42 days of termination of pregnancy". ${ }^{2}$ This study is aimed in evaluating incidence, nature of near miss and the quality of measures undertaken in a tertiary hospital. This is done through the standard indicators- severe maternal outcome ratio (SMOR), maternal near-miss ratio (MNMR), maternal near-miss mortality ratio (MNM: 1MD) and mortality Index (MI).

\section{Subjects and methods}

An observational study of maternal near-miss cases from June 2012 to May 2013, in Kasturba Hospital, Manipal University, Manipal. It is a tertiary referral hospital for Udupi and 3 other districts in Karnataka, India.
The following steps were followed in collecting cases.

a. Identification of potentially life-threatening conditions: Patients who had complications at the time of pregnancy and delivery or who were referred with complications were identified from the maternity record that we maintain in our department.

b. Classification as near- miss cases: Case records of all the patients who had had potentially life threatening complications were analyzed and near- miss cases were classified according to 2009 WHO criteria. ${ }^{3}$

c. All required details about maternal deaths were also collected.

After collection of the details, compilation of it is done and the various indicators of severe acute maternal mortality were calculated. These are:

A. Severe maternal outcome ratio (SMOR) which is number of women with life threatening conditions per 1000 live births (MNM+MD/ LB).

B. Maternal near-miss ratio (MNMR) which is number of maternal near miss cases per 1000 live births (MNM/ LB).

C. Maternal near-miss mortality ratio (MNM: $1 \mathrm{MD}$ ) defined as ratio between maternal near miss cases and maternal deaths (MNM: $\mathrm{MD})$.

D. Mortality Index (MI)- Number of maternal deaths divided by number of women with life threatening conditions (MD/ $\mathrm{MNM}+\mathrm{MD})$. 


\section{Results}

During the study period, there were 1495 live births among which 335 mothers had potentially life-threatening complications. On analyzing, near-miss cases were found to be 64 and 10 among them ended up as maternal mortality (Table 1).

Table I The demographic characteristics of the near miss patients and maternal deaths

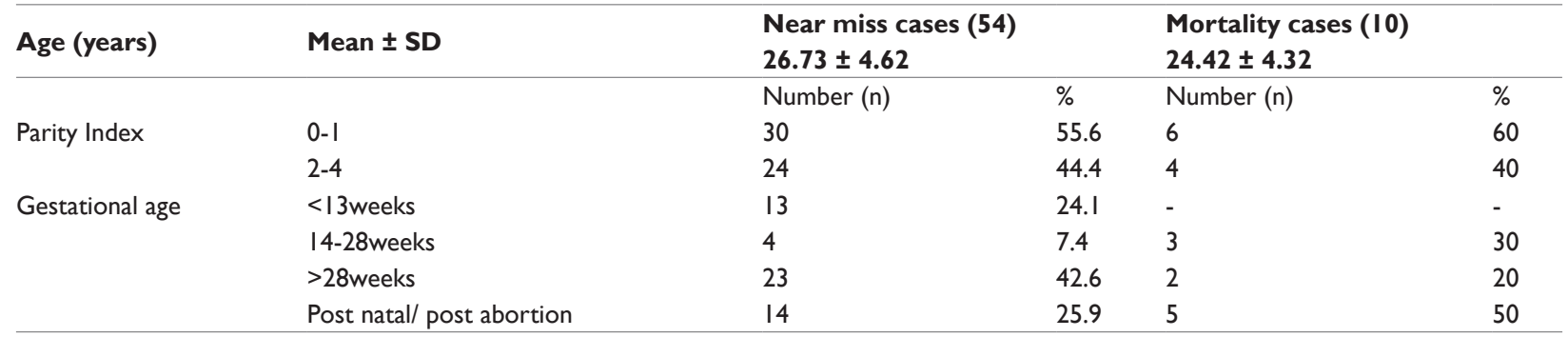

Primigravidae are numerically more in the near miss group, but it was not statistically significant. Incidence of near miss events were more among the mothers in the third trimester $(42.66 \%)$ whereas maternal mortality was high among the post-natal mothers, including both post delivery as well as post-abortal patients- the most common cause being puerperal sepsis. Among the 64 near miss cases, patients who developed life -threatening conditions within hospital were 12 and the rest 52 patients were referred from elsewhere. All the 52 referred patients were fitting in the near-miss criteria at the time of admission (Table 2).
Post-partum hemorrhage accounts for most of the near-miss cases, with a near-miss ratio of $27 \%$. Septicemia has the highest mortality index (42\%) apart from miscellaneous causes like primary pulmonary hypertension and gestational trophoblastic disease with metastasis. Among the patients who fit into the near-miss criteria, 47 of them required blood product transfusions (including PRBC, FFP, Platelets and cryoprecipitates), 4 patients underwent Uterine artery embolization (radiological intervention), 28 patients had laparotomy (excluding cesareans), and 29 patients required admission under intensive care unit. Coagulation dysfunction is the most common organ dysfunction noted among near-miss cases (Figure 1).

Table 2 The near miss mortality ratio and mortality index of various complications (outcome)

\begin{tabular}{|c|c|c|c|c|}
\hline Complication & Near miss (n) & Mortality (n) & $\begin{array}{l}\text { Near miss ratio } \\
\text { (per I000 LB) }\end{array}$ & $\begin{array}{l}\text { Mortality } \\
\text { index (\%) }\end{array}$ \\
\hline Post partum hemorrhage & 17 & 2 & 27 & II \\
\hline Pre- eclampsia & 8 & 0 & 12 & - \\
\hline Eclampsia & 4 & 0 & 6 & - \\
\hline Septicemia & 7 & 5 & II & 42 \\
\hline Rupture uterus & 6 & 0 & 9 & - \\
\hline $\begin{array}{l}\text { Ectopic gestation } \\
\text { with complications }\end{array}$ & 12 & 0 & 19 & - \\
\hline $\begin{array}{l}\text { Others (Pulmonary artery } \\
\text { hypertension, metastasis) }\end{array}$ & 0 & 3 & & 100 \\
\hline
\end{tabular}

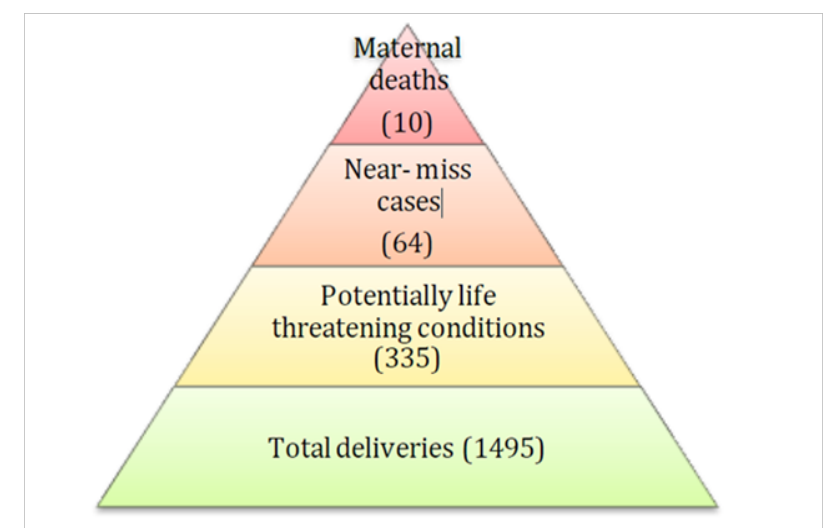

Figure I Near-miss pyramid- Incidence in the study population.

The calculated severe maternal Outcome ratio (SMOR) is 43 per I000live births, maternal near-miss ratio (MNMR) of 36 per 1000 live births, Near-miss mortality ratio of $5.4:$ I and mortality index (MI) of $15.6 \%$.

\section{Discussion}

In our institution the most common cause of maternal morbidity is post-partum hemorrhage $(26.65 \%)$ followed by hypertensive disorders $(18.7 \%)$ which was similar to most of the previous studies. ${ }^{4}$ Septicemia was the leading cause of maternal mortality $(50 \%)$ in the study.

The maternal near-miss ratio of our institute of 36 per 1000 live births was within the range of MNMR reported among the developing countries- 12.3 to 82.3 per 1000 live births. ${ }^{5}$ These studies also used the similar WHO criteria to define maternal near-miss cases.

Near Miss Cases were identified according to 2009 WHO criteria, ${ }^{3}$ which includes clinical, laboratory-based and management-based criteria. The most easily interpreted one among the 3 was clinical criteria and it was used for retrospective analysis without difficulty. Laboratory-based criteria was considered as the most specific, draw back being it is difficult to obtain all the investigations done in a low resource setting. ${ }^{6}$ But this did not arise as a problem in our set up which is a tertiary care centre. Among the patients who were identified 
with severe acute morbidity, $81.25 \%$ developed it on arrival to the hospital (referred patients). This reflected the serious lack in timely referrals and problems in transportation facilities. A similar problem was reported in one of the studies in Syria(7), that stated $31 \%$ of maternal deaths were among home deliveries whereas $17 \%$ of them were hospital deliveries and were shifted to other hospitals because of lack of facilities. The other socioeconomic factors that influence the severe acute maternal morbidity are low socio economic status where people tend to delay in seeking medical help. Similar reports were reported in a study carried out in Uganda. ${ }^{8}$

The most important limitation of this study is that it is a retrospective study done over a short duration of one year. Thus the numericals and statistics could not reflect the actual problem load and it cannot be compared with the national statistics. But a declining trend of mortality index and MNMR are noted in our institute when compared with the previous similar study undertaken in our institute. ${ }^{9}$

The incorporation of near-miss cases into maternal death enquiries would strengthen these audits by allowing more rapid reporting, comparison with maternal deaths, reinforcing lessons learnt and establishing requirements for intensive care. The survival of pregnant woman is dependent on the disease, her basic health, the health care facilities and the personnel of health care system. Hence, near-miss events must be estimated separately for those already in a critical condition on arrival and those developing complications after admission; the first as a good indicator of the effectiveness of referral systems and the second defining a potential pattern of maternal death.

\section{Conclusion}

Post-partum haemorrhage accounted for maximum number of near-miss cases whereas septicaemia stands as the leading cause of maternal mortality. There was no mortality attributed to hypertensive disorders of pregnancy. Though maternal near-miss is unpredictable, anticipatory and active management at the appropriate time can reduce maternal mortality. Every health care personnel have an opportunity to improvise their delivery of health care and such audits help in identifying the area to be improvised. The incidence of near-miss also depends on multiple confounding factors like first referral service, transport facility, knowledge and awareness among community in which media also should play a role in creating awareness.

\section{Acknowledgments}

None.

\section{Conflicts of interest}

None.

\section{References}

1. UNFPA. Trends in Maternal Mortality: 1990-2010. World health Organization, Switzerland. 2012.

2. Say L, Souza JP, Pattinson RC. Maternal near miss towards a standard tool for monitoring quality of maternal health care. Best Pract Res Clin Obstet Gynaecol. 2009;23(3):287-296.

3. Pattinson R, Say L, Souza JP, et al. WHO maternal death and near-miss classifications. Bull World Health Organ. 2009;87(10):734.

4. Jayaratnam S, De Costa C, Howat P. Developing an assessment tool for maternal morbidity "near-miss"-a prospective study in a largeAustralian regional hospital. Aust N Z J Obstet Gynaecol. 2011;51(5):421-425.

5. Say L, Pattinson RC, Gülmezoglu AM. WHO systematic review of maternal morbidity and mortality: the prevalence of severe acute maternal morbidity (near miss). Reprod Health. 2014;1(1):3.

6. Ronsmans C, Filippi V. Reviewing severe maternal morbidity: learning from women who survive life threatening complications. In: Lewis G (Ed.), Beyond the Numbers Reviewing Maternal Deaths and Complications to Make Pregnancy Safer. Geneva, Switzerland: World Health Organization. 2004;pp.103-123.

7. Bashour H, Abdulsalam A, Jabr A, et al. Maternal Mortality in Syria: Causes, contributing factors, and preventability. Trop Med Int Health. 2009;14(9):1122-1127.

8. Weeks A, Lavender T, Nazziwa E, et al. Personal accounts of nearmiss maternal mortalities in Kampala, Uganda. Br J Obstet Gyneacol. 2005;112(9):1302-1307.

9. Ps R, Verma S, Rai L, et al. Near Miss" Obstetric Events and Maternal Deaths in a Tertiary Care Hospital: An Audit. J Pregnancy. 2013:393758. 